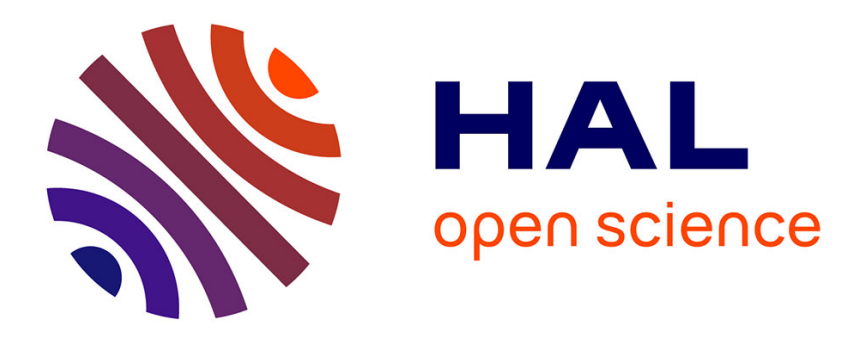

\title{
Integrating PPI Variability in the Context of Customizable Processes by Extending the Business Process Feature Model
}

\author{
Diaz Diego
}

\section{- To cite this version:}

Diaz Diego. Integrating PPI Variability in the Context of Customizable Processes by Extending the Business Process Feature Model. EDOC 2020, Oct 2020, Eindhoven (on line), Netherlands. hal02946194

\section{HAL Id: hal-02946194 \\ https://hal.science/hal-02946194}

Submitted on 29 Sep 2020

HAL is a multi-disciplinary open access archive for the deposit and dissemination of scientific research documents, whether they are published or not. The documents may come from teaching and research institutions in France or abroad, or from public or private research centers.
L'archive ouverte pluridisciplinaire HAL, est destinée au dépôt et à la diffusion de documents scientifiques de niveau recherche, publiés ou non, émanant des établissements d'enseignement et de recherche français ou étrangers, des laboratoires publics ou privés. 


\title{
Integrating PPI Variability in the Context of Customizable Processes by Extending the Business Process Feature Model
}

\author{
Diaz Diego \\ Univ. Grenoble Alpes, CNRS, Grenoble INP, LIG, \\ 38000 Grenoble, France \\ diego.diaz@univ-grenoble-alpes.fr
}

\begin{abstract}
While model support concerning the variability and customization of business processes has been studied extensively, related Process Performance Indicators (PPIs) have not been addressed similarly. An approach that helps PPI calculation and promotes reusability in this context is critical for organizations. A fast adaptation of their information system is needed, concerning new clients' requirements, new policies or evaluations of audit entities. Business Process Feature Model (BPFM) is a well-known approach to support process variability by modeling all possible variants and generating process skeleton based on different customizations. However, this approach does not consider the PPI's modeling and calculation variability. In this paper, we will talk about the thesis project concerning the extension of the BPFM approach in order to the integrate PPI variability. We present our first results relying on our experience in a real industrial case; a tree model of PPIs variability linked to the BPFM model facilitating the modeling, the customization and the reasoning about PPIs variants depending on the process customization and PPIs definitions.
\end{abstract}

Keywords- Process Performance Indicators, Process Families, Variability

\section{THE CONTEXT AND GOAL OF THE RESEARCH}

While model support concerning the variability and customization of Business Processes (BP) has been studied extensively [1][2], related Process Performance Indicators (PPIs) have not been addressed similarly [3][4][5]. Organizations identify PPIs in order to provide the necessary data to decision-makers to compare current process performances with a required objective, so that decision-makers can determine necessary actions and reach proposed goals [6]. Indeed, organizations must adapt their information system, and thus their process and PPIs, to new clients' requirements, new policies or audit entities evaluations. The support of process variability makes PPIs' definition and calculation difficult, which is tightly related on the process [7]. The creation of new PPIs is also a time-consuming and error prone task, highly dependent on the expert know-how, who does not easily have the visibility of the customizable process. Therefore, an approach that helps PPI calculation and promotes reusability in this context is critical.

This paper focuses on the PPI modeling and calculation in the context of customizable processes. Domains such as Business Process Model Families (BPMFs) [8] and PPI modeling [9] respond in part to our need. For instance, the Business Process Feature Model (BPFM) [10] is an approach to model process families extending the Feature Models, which is a classic representation of software product lines variability [11] [12]. However, process family's models and languages such as BPFM do not support PPI variability. Likewise, the approaches modeling PPI variability such as PPINOT [9] are not integrated with customizable process, as they treat PPI variability in the context of a predefined process model.

In the context of this $\mathrm{PhD}$ thesis, we tackle variability in both directions by extending the BPFM approach with PPI variability based on our experience in a real industrial case. Our practical use case concerns public administrations in the context of utilities distributors, that rely on software publishers such as INCOM to implement BP and PPI required by utilities' decision-makers and regulation entities, which may have different requirements concerning the same utility distribution. Our approach proposes a PPI tree linked to the BPFM with a predefined calculation following certain construction rules. The PPI tree might be extended by designers to create new PPIs relying on the generated skeleton. This integration facilitates the modeling, the customization and the reasoning about PPI variants depending of process customizations.

The rest of the paper is structured as follows. Section 2 reviews related works. Section 3 presents our approach that integrates PPIs within BPFM as well as our use case. Finally, section 4 concludes, summarizes and presents the considered perspectives.

\section{THE SCIENTIFIC PROBLEM ADDRESSED IN THE PHD PROJECT}

\section{A. Context and research problem}

Our research problem emerges from the industrial of INCOM, a French software publisher that works for 230 utility distributors supporting their business management. Every distributor evaluates its own processes under different criteria. During this year and a half of CIFRE PhD thesis (industrialresearch agreement between a company and a research center), we have set the following research questions regarding INCOM requirements: I) how to integrate the PPIs into the business processes families?, II) how to model and calculate the PPIs variables in the context of business processes families? and III) how to recalculate an existing PPI or calculate a new one according to customizable processes? 
We illustrate the PPI variability through one of the most common INCOM's clients process called Create Contract. INCOM customizes and deploys independently this process for every water distributor and must provide them relevant indicators regarding stakeholders' requirements, cf. Fig. 1. Every variant of Create Contract is a customization of the process model due to the client's internal organization and certain general rules adapted to their specific know-how.

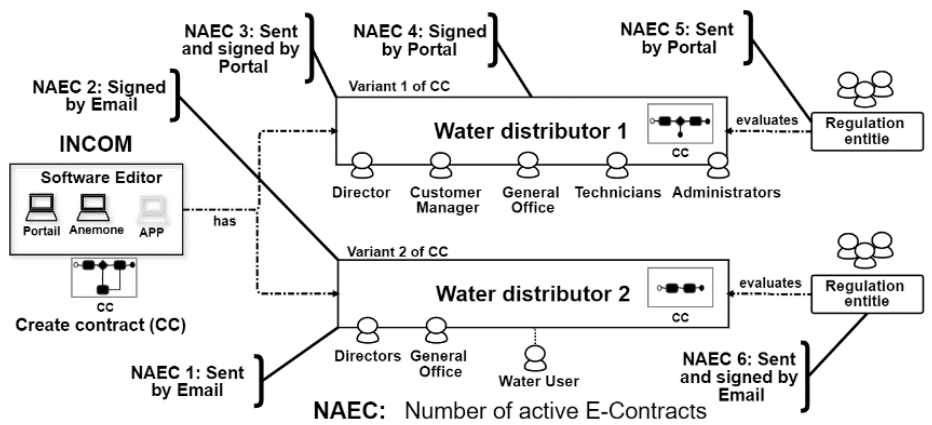

Figure 1 PPI Variability within the Water Distributor context

The same PPI can have several variants depending on a specific context. For instance, in Fig. 1 for the PPI Number of active E-Contracts (NAEC) a stakeholder may actually want to calculate the Number of active Signed Contracts by Email (NAEC 2). Another client may be interested in the Number of active Sent and Signed Contracts by the Portal (NAEC 3), while an audit entity will require the Number of active Sent and Signed Contracts by Email (NAEC 6). Thus, we distinguish two PPI variants types: I) PPIs derived of client's customized process models, i.e., all PPI linked to the process customization, e.g., if the process uses Portal (NAEC 3, 4 and 5) or Email (NAEC 1, 2 and 6), and II) PPIs derived of stakeholders' definitions, i.e., all PPI linked to business definitions (NAEC 1 or 2 or 3 ).

\section{B. Identified conditions for the Ph.D research problem}

In order to implement PPIs in a process variability approach, we have identified 7 fundamental conditions relying on our industrial case experience and our research needs. Requirements: The approach must I) support variability by restriction to model and calculate PPIs for known process variants, II) support variability by extension to model and calculate PPIs for process variants not known a priori, III) support objects, specifically data-objects, to extract necessary information to calculate PPIs, IV) have an exploitable and extensible implementation to include the calculation of variable PPIs. According to [3], the performance perspective of BPs is subject to variation like other perspectives and, as such, it is convenient to develop models and tools that manage this variability, favor reuse and reduce design and maintenance time; constraints: V) be validated, preferably through study cases in public administrations, VI) avoid as much as possible behavioral anomalies such as deadlocks and livelocks when the model is instantiated, VII) guide users as much as possible when making customization decisions to select one option or another, that is, prevent users from making inconsistent or irrelevant customization decisions from the domain angle.

\section{RELATED WORKS}

As mentioned before, variability and customization of processes models has been studied extensively. This customization is divided in two types of variability management: by restriction and by extension [1]. Variability by restriction refers to a customizable process model that contains all the behavior of all process variants. Customization is accomplished by restricting the behavior of the customizable process model, e.g., activities may be skipped or blocked during customization [1]. Customizable process models of this type are also called configurable process models, defined as "the ability to express and produce different variants of a process from a configurable model" [13]. Some approaches that support this variability are PESOA [14], C-YAWL [15] and C-iEPC [8] among others. Variability by extension refers to a customizable process model that does not contain all possible behavior; instead, it represents the most common behavior in process variants. During customization the model's behavior is extended for a specific context, e.g., new activities may be inserted to create a dedicated variant [1]. Some examples are PROVOP [13], ADOM [16] and vBPMN [17] among others.

After evaluating the approaches regarding our desired conditions showed in the previous section, we note that none of them meets all the requirements and constraints. The closest approaches are Configurative Process Modeling, PROVOP and Template and Rules [1] meeting 12 out of 14 criteria Fig 2. However, there is an approach that has not been analyzed by [1] Business Process Feature Model (BPFM) [10], which includes refinement variants even if the process has been customized Fig 2. It also considers the deployment context information adapting execution paths for every process variant Fig 2. Moreover, implemented in the ADOxx platform, thus BPFM is executable and allows to guide users to make customization decisions and avoid behavioral anomalies for every process variant Fig 2. Likewise, BPFM supports data-objects allowing to obtain generated information by each activity, which serves as a guide to calculate desired PPIs Fig 2. Finally, this approach has been validated considering several Public Administration scenarios through the European Project Budget Report case study endorsed by the Learn PAd Project.

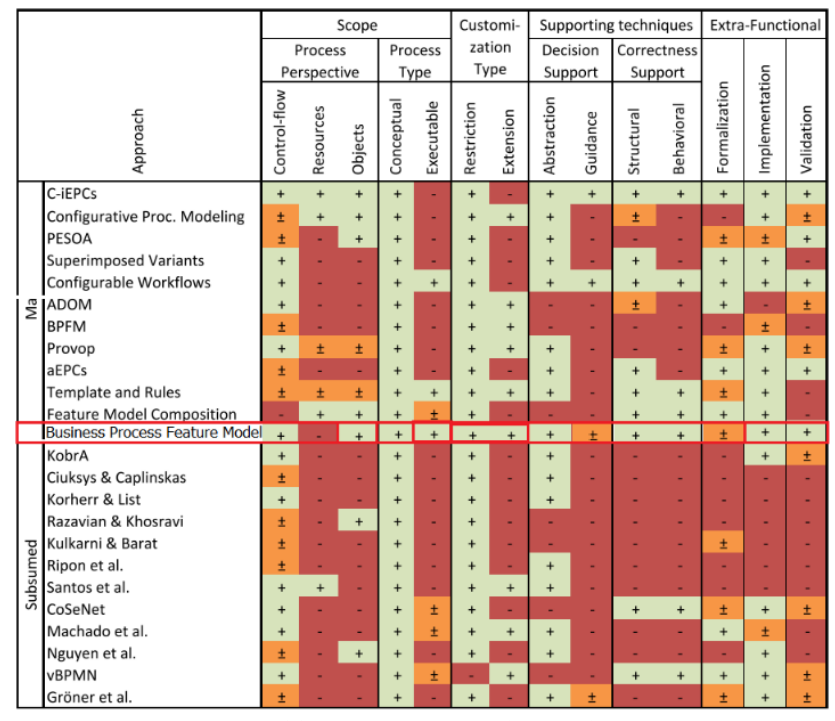

Figure 2 Customizable process approaches evaluation 
PPIs' variability has not been addressed so extensively in the literature [3][18]. For instance, classical architectures such as Data Warehouse, Business Intelligence, Business Activity Monitoring [19], Business Performance Management [20] or modeling performance indicators [21] deal with the importance of enforcing objectives defined by business strategies and metrics to model and calculate indicator. Nevertheless, in the case of BP families, information extraction from business data is not enough, especially when different PPI definitions depend on flexible evaluation criteria and customized process models. The PPIs variability [3] allows an advanced definition of variable performance indicator independently of the language used to model the BP. However, it does not consider customizable process models based on variability by extension. The PPINOT approach [9] proposes a language for defining and modeling PPIs variability together with business process. Nevertheless, it also does not consider customizable process models. Likewise, neither [3] nor [9] allow to model and define relations between PPIs for customizable process models.

From the study of the state of the art, we conclude that BPMFs seek to partly respond to the modeling problematic when an organization adopts new BPs' activities. However, BPMFs have not been designed to determine the impact of process variability in the PPIs calculation. Thus, when an organization explores its data sources and uses it as part of new process, there are no business-related PPI lists. To propose candidate PPIs, managers must rely on their intuition, which causes some PPIs to be redundant, increasing the necessary efforts and resources in its calculation [22]. Our approach extends BPMFs to guide and facilitate the construction of new PPIs from existing ones inspired by the PPINOT approach to cover the PPI variability concern.

\section{SCIENTIFIC METHOD}

We rely on the THEDRE method since it allows to lead researches in human-centered computer science and guide researchers in the selection of a user-centered experimental method [23]. This method is a formalized process to manage research with tools from quality management and allows its actors, e.g., researchers, developers and methodologists, guidance at each step of the research process. THEDRE has been structured according to the continuous quality improvement cycle: Plan Do Check Act (PDCA): Plan for the research plan, Do to guide developments and conducting experiments, Check for assessing results and Act, which allows researchers to construct knowledge regarding assessments and to take the decision to start a new cycle or to communicate the results (ex., by writing a paper). We illustrate these four steps through our thesis project as follows:

PLAN: in this stage we have focused on research construction and we have set three research targets to achieve concerning developments, experiments, and communications. During the first cycle, we have set the following research questions: I) how to integrate the PPIs into the business processes families?, II) how to model and calculate the PPIs variables into the business processes families?, and, III) how to recalculate an existing PPI or calculate a new one according to deployed processes into the business processes families?. Likewise, during the first cycle we have set the experimental targets like I) involve indicator developers in the modelling of PPIs according to customizable processes, II) explore how users express their PPIs definitions, and III) identify users' modeling methodologies and calculation practices of PPIs.

DO: in this stage we have focused on the development of experiments to assess the main concepts of our research instrument. For the first cycle, three experiments with experts were realized to validate the model proposed concerned the creation of a process performances indicators modelling language. During this qualitative experimental protocol, each section had work and reflection scenarios intercalated and supported by interview guides. Thus, experts expressed their views individually on either improvements or questions.

CHECK: in this stage we have assessed the experimental results and controlling targets. Some research targets were checked such as methodological hypotheses and clarity of concepts. This step was crucial to reason about the following experiments and control the thesis progress and course.

ACT: during this stage, we have attained some scientific knowledge from the experimental results and its limits. Likewise, we are now reasoning about the next PCDA cycle of to experiment with a group of novices. Likewise, we are documenting the results to communicate our progress. For now, according to our analysis we must perform another PCDA research cycle to improve our model and method proposing a modeling tool for PPIs variability into business process families.

\section{CURRENT RESUlTS: SUPPORTING PPI VARIABILITY IN THE CONTEXT OF PROCESS FAMILIES}

\section{A. The Process Performance Indicator Calculation Tree (PPICT) definitions}

The PPICT can be defined as an approach to model variables PPIs as a tree and facilitate its calculation and reuse in the context of customizable process. PPICT relies on BPMFs definitions, proposing the term family of PPIs following the same pattern as the family of processes in the case of customizable process model [1]. Therefore, we define a family of PPIs as "a paradigm for calculating PPIs using a set of processes and subsystems that form a common structure, which serves as a basis for derived PPIs and it is adapted to each client needs", i.e., a family of PPIs refers to the calculation variability of a set of PPIs sharing a certain number of operators, e.g., projections, selections, joins and relations, since we use a query tree of relational algebra to illustrate PPIs, Fig. 3. Due to these shared similarities, the PPI families' development improves the reuse and profitability of decision reports corresponding to the customized process models. Additionally, a family of PPIs is linked to a family of processes to measure and evaluate only desired processes by stakeholders.

To model a family of PPIs, we propose the Process Performance Indicator Calculation Tree (PPICT), which provide a global representation of PPIs calculation in a given domain through the systematic modeling of variability and common points of recurrent PPIs, i.e., the PPICT define the available PPIs members of a family of PPIs, as well as dependencies between them. The PPIs are described as a tree, where each entity represents a PPI from the set of PPIs available in a decision report. The PPICT approach aims at combining BP families and 


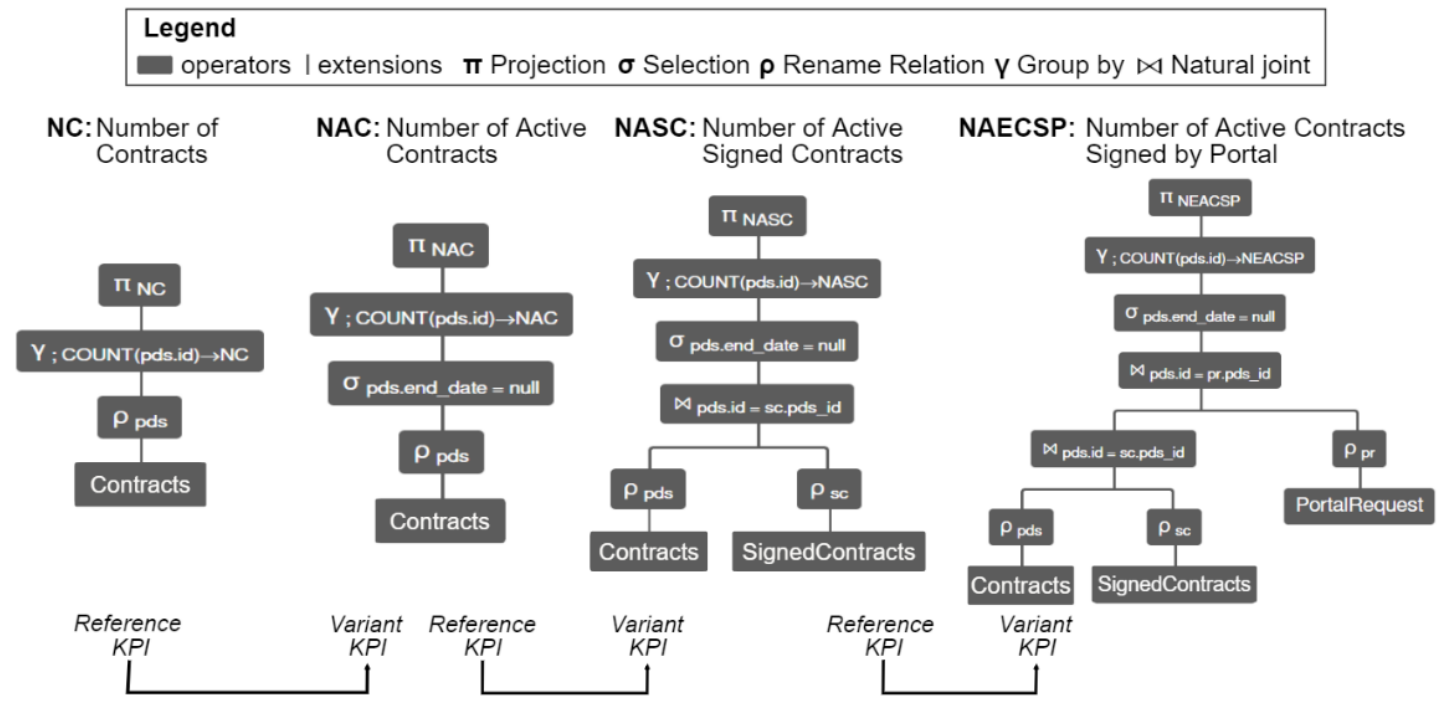

Figure 3 Query tree and PPI relationship

PPIs families modeling relying on the BPFM. Below proposed definitions to model PPIs families through the PPICT approach. We extend the PPIs definitions proposed by [3] including relations between PPIs and process activities:

Process Performance Indicator (PPI) can be defined as a quantifiable metric focused on evaluating the performance of a $\mathrm{BP}$ in terms of efficiency and effectiveness [3]. They are measured directly by data generated within the process flow and are used for process controlling and continuous optimization [18]. Likewise, a PPI is a set of tuples represented either as a value, a percentage, a list, a map or a chart. This set is a measure required and regulated by stakeholders that manage and evaluate the process performance. This set also must be computed regarding a calculation type that is detailed later. The result of these operations is called resulting tuples. A PPI can be calculated differently depending on I) desired process model customization, II) definition of metrics by stakeholders, III) activities involved. A PPI can be illustrated using a query of relational algebra, whose nodes are operators and arcs are extensions, i.e. relationships between operators, Fig. 2 .

Reference PPI is a PPI that serves as the basis for calculating its variant PPIs, e.g. Fig. 1 shows that the Number of Contracts $\mathrm{NC}$ is the reference PPI of the Number of Actives contracts NAC, which added the operator $\sigma$ pds. end_date = null to the original query of NC. NAC is the reference PPI of NASC, which added the operators $\rho s c$ and $\rho s c$ (SignedContracts). It means that every variant PPI only adds operators to the original query of its reference PPI. i.e., a variant PPI does not delete any operators from its reference PPI. The operators that can have a reference PPI are projections, selections, relations, rename, intersections, difference, joins, duplicate elimination, grouping and aggregation. Likewise, any of these operators can be added in a variant PPI if resulting tuples of a reference PPI contain all the possible resulting tuples of its variant PPIs, i.e. all resulting tuples of variant PPIs must be a subset of resulting tuples of its reference PPI.

Variant PPI is a PPI derived from its reference PPI. A variant PPI contains at least: I) the same projections than its reference, II) the same joins than its reference, III) the same relations than its reference, and, IV) the same selections than its reference. A variant PPI doesn't delete any operators from its reference PPI. A variant PPI can have a different graphical representation than its reference PPI and its siblings derived from the same reference, i.e., a new representation of the reference PPI implies a new variant PPI, e.g., if the reference PPI's representation is a value and we want the percentage, we must calculate a new variant PPI. A variant PPI allows to add the operators already described in the reference PPI definition. Additionally, all resulting tuples of variant PPIs must be contained among resulting tuples of its reference PPI. This means that a variant PPI must have only one reference PPI that meets this condition.

PPICT model is a set of PPIs organized in a tree, where the tree's root identifies a family of PPIs. Each PPI of the internal tree's structure is a reference PPI, i.e., each PPI that is not a tree leaf is a reference PPI including the root. Regarding PPIs-leaf, they are variants of a higher-level PPI. Additionally, all PPIs of the internal structure except for the PPI-root are also variants of a higher-level PPI, i.e., the only PPIs that have a single role are the PPIs-leaf with variant role and the PPI-root with the reference role. Additionally, the PPICT model allows to include different calculation detail levels as PPI specifications and as the BPFM approach, connections between reference and variant are called constraints. These constraints are detailed in the next section.

\section{B. Constraints linking PPICT with BPFM}

The rules that guide the integration between BPFM and the PPI Tree are presented here. The PPICT's constraints are divided into 3 groups: Binary Constraint, Multiple Constraint and PPI-Activity Constraint. Theses Constraints represent I) dependencies between reference PPIs and variant PPIs, II) associations between PPIs and activities. Every individual variant PPI added to the tree must be connected to its reference PPI as a binary relationship. A PPI can be the reference of several individual variants, but a variant has only one reference. Below the PPICT Binary Constraint is defined: 


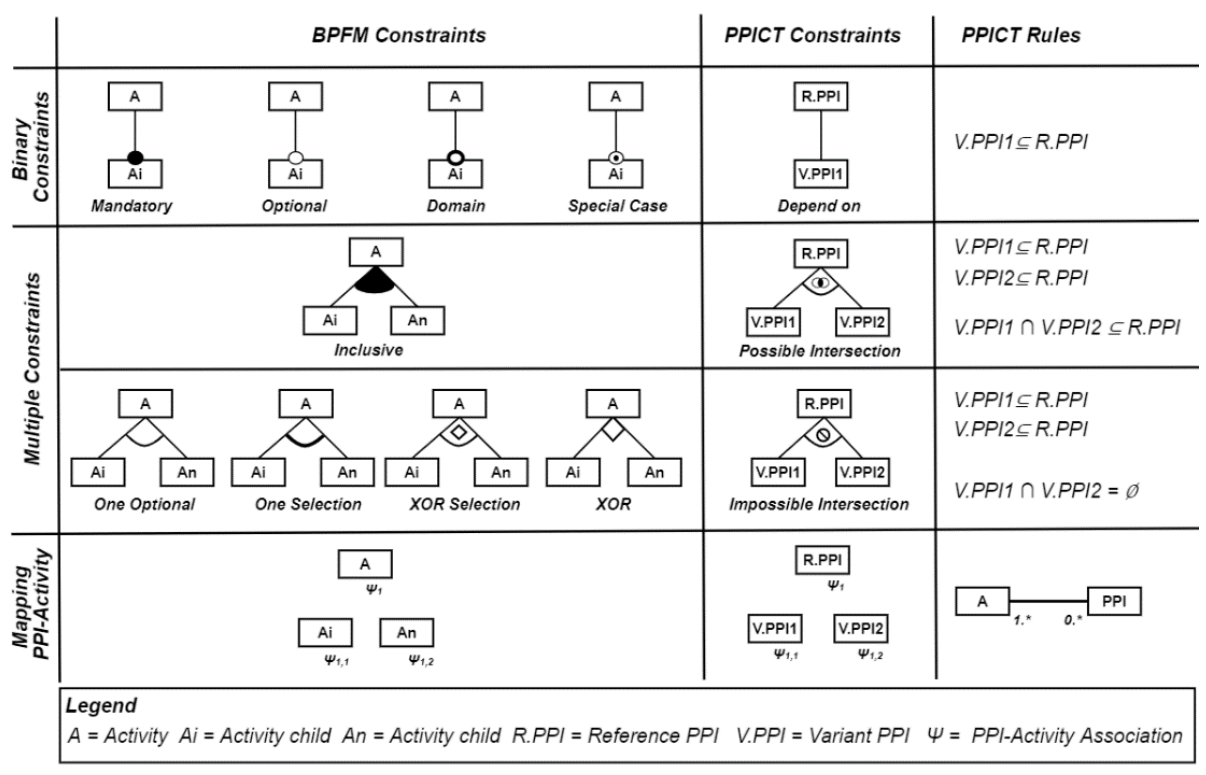

Figure 4 PPICT's Constraints

- A Depend-on Constraint specifies that all resulting tuples of the connected variant PPI are a subset or equal to resulting tuples of its reference PPI (cf. Fig. 4).

Every group of variant PPIs added to the tree must be connected to its reference PPI as multiple relationship. For this we propose PPICT Multiple Constraints, which specify the dependency between a PPI and a group of PPIs to determine their role into the tree. A PPI can be the reference of a group of variants, but each variant must have only one reference. Below the PPICT Multiple Constraints are defined:

- An Overlap Constraint specifies that all intersections between variant PPIs are a subset or equal to resulting tuples of its reference PPI (cf. Fig. 4).

- A Disjoint Constraint specifies that all intersections between variant PPIs are equal to zero $\emptyset$, i.e. all intersections between variant PPIs are disjoint sets (cf. Fig. 4).

Since PPICT is an extension of the BPFM approach, it is necessary to link the PPI families modeling and the BP families modeling. In this sense, we propose the PPI-Activity association, which defines that an activity can have zero or several associated PPIs and that a PPI must have at least one associated activity to be calculated:

- PPI-Activity Constraint refers to the association of a PPI with at least one activity of the BP family, i.e., BPFM Model (cf. Fig. 4).

Essentially, PPICT's constraints allow to associate PPIs to the BPFM model, following some construction properties. The next section presents an example of PPICT implementation concerning our industrial case.

\section{PLAN FOR THE EVALUATION OF RESULTS AND EXPECTED RESULTS}

The first qualitative experimentations cycle allowed us to measure the PPI development improvements within INCOM's engineers when using the approach, e.g., experts mentioned the complexity of controlling process' changes without proper modeling, induce that the PPIs be recalculated. Further, experts agree that currently there is a lack of reliability in the calculation of PPIs because they do not have tools or methods that allow them to prune and calculate PPIs. That is why, an evolution of PPICT approach is the formalization of a method to guide the use of this model starting from a pre-defined BP family. Likewise, a metamodel must be implemented and validated in order to align the approach according to PPICT's constraints, cf. Fig 4. As future research activity, we plan also to implement this method by extending the BPFM tool which is already hosted on the ADOxx metamodeling platform contributing in this way to OMiLAB community. This implementation should be used an appropriate method by practitioners involving PPIs developers and decision makers.

Otherwise, some limitations that experts evoked and that should be improved in futures research activities are: "There is no view of the query on the constraints diagram", "Classify a PPI in a family too large is without doubt difficult", "Adding new relation can be complicated if we don't know a little bit the data", "The terminology is a little bit complicated; father and son can be an alternative for reference and variant PPI".

\section{CONCLUSION}

The PPIs variability support has less been treated than the process variability support. However, the support of process variability, complicates the PPIs' definition and calculation, which is tightly dependent on the process customization. In the context of this PhD thesis we explore the PPICT approach, relying on our industrial experience as software publisher for public administrations. We extend the BPFM approach integrating PPI variability within the BP families. The solution aims at facilitating the creation and reasoning of PPIs in the context of BP families. 


\section{REFERENCES}

[1] La Rosa, Marcello, W. M. P. Van Der Aalst, M. Dumas, and F. P. Milani, "Business process variability modeling: A survey," ACM Comput. Surv., vol. 50, no. 1, p. 2, 2017.

[2] F. Milani, M. Dumas, N. Ahmed, and R. Matulevičius, "Modelling families of business process variants: A decomposition driven method," Inf. Syst., vol. 56, pp. 55-72, 2016.

[3] B. Estrada-Torres, A. Del-Río-Ortega, M. Resinas, and A. Ruiz-Cortés, "Identifying variability in process performance indicators," in International Conference on Business Process Management, 2016, pp. 91-107.

[4] E. Domínguez, B. Pérez, Á. L. Rubio, and M. A. Zapata, "A taxonomy for key performance indicators management," Comput. Stand. Interfaces, vol. 64, pp. 24-40, 2019.

[5] B. Estrada-Torres, "Improve Performance Management in Flexible Business Processes," in Proceedings of the 21st International Systems and Software Product Line Conference-Volume B, 2017, pp. 145-149.

[6] J. Peral, A. Maté, and M. Marco, “Application of data mining techniques to identify relevant key performance indicators," Comput. Stand. Interfaces, vol. 54, pp. 7685, 2017.

[7] B. Estrada Torres, V. Torres, A. del Río Ortega, M. Resinas Arias de Reyna, V. Pelechano, and A. Ruiz Cortés, "Defining PPIs for Process Variants based on Change Patterns," JCIS 2016 XII Jornadas Cienc. e Ing. Serv. (2016), 2016.

[8] M. La Rosa, M. Dumas, A. H. M. Ter Hofstede, and J. Mendling, "Configurable multi-perspective business process models," Inf. Syst., vol. 36, no. 2, pp. 313-340, 2011.

[9] A. del-Río-Ortega, M. Resinas, A. Durán, B. Bernárdez, A. Ruiz-Cortés, and M. Toro, "Visual PPINOT: A graphical notation for process performance indicators," Bus. Inf. Syst. Eng., vol. 61, no. 2, pp. 137-161, 2019.

[10] R. Cognini, F. Corradini, A. Polini, and B. Re, "Business process feature model: an approach to deal with variability of business processes," in DomainSpecific Conceptual Modeling, Springer, 2016, pp. 171194.

[11] G. Gröner et al., "Validation of families of business processes," in International Conference on Advanced Information Systems Engineering, 2011, pp. 551-565.

[12] A. Villota, R. Mazo, and C. Salinesi, "The high-level variability language: an ontological approach," in Proceedings of the 23rd International Systems and Software Product Line Conference-Volume B, 2019, pp. 162-169.
M. Reichert, A. Hallerbach, and T. Bauer, "Lifecycle management of business process variants," in Handbook on Business Process Management 1, Springer, 2015, pp. 251-278.

[14] A. Schnieders and F. Puhlmann, "Variability Mechanisms in E-Business Process Families.," in Proceedings of the 9th International Conference on Business Information Systems (BIS'06), 2006, vol. 85, pp. 583-601.

[15] F. Gottschalk, T. A. C. Wagemakers, M. H. JansenVullers, W. M. P. van der Aalst, and M. La Rosa, "Configurable process models: Experiences from a municipality case study," in International Conference on Advanced Information Systems Engineering, 2009, pp. 486-500.

[16] I. Reinhartz-Berger, P. Soffer, and A. Sturm, "Organisational reference models: Supporting an adequate design of local business processes," Int. J. Bus. Process Integr. Manag., vol. 4, no. 2, pp. 134-149, 2009.

[17] M. Döhring and B. Zimmermann, "vBPMN: eventaware workflow variants by weaving BPMN2 and business rules," in Enterprise, Business-Process and Information Systems Modeling, Springer, 2011, pp. 332-341.

[18] A. Del-RíO-Ortega, M. Resinas, C. Cabanillas, and A. Ruiz-Cortés, "On the definition and design-time analysis of process performance indicators," Inf. Syst., vol. 38, no. 4, pp. 470-490, 2013.

[19] J.-P. Friedenstab, C. Janiesch, M. Matzner, and O. Muller, "Extending BPMN for business activity monitoring," in 2012 45th Hawaii International Conference on System Sciences, 2012, pp. 4158-4167.

[20] M. Golfarelli, S. Rizzi, and I. Cella, "Beyond data warehousing: what's next in business intelligence?," in Proceedings of the 7th ACM international workshop on Data warehousing and OLAP, 2004, pp. 1-6.

[21] V. Popova and A. Sharpanskykh, "Modeling organizational performance indicators," Inf. Syst., vol. 35, no. 4, pp. 505-527, 2010.

[22] R. R. Rodriguez, J. J. A. Saiz, and A. O. Bas, "Quantitative relationships between key performance indicators for supporting decision-making processes," Comput. Ind., vol. 60, no. 2, pp. 104-113, 2009.

[23] N. Mandran and S. Dupuy-Chessa, "Supporting experimental methods in information system research," in 2018 12th International Conference on Research Challenges in Information Science (RCIS), 2018, pp. 112. 